\title{
Primer extension based quantitative polymerase chain reaction reveals consistent differences in the methylation status of the MGMT promoter in diffusely infiltrating gliomas (WHO grade II-IV) of adults
}

\author{
Erik Vassella $\cdot$ Istvan Vajtai $\cdot$ Nora Bandi $\cdot$ \\ Marlene Arnold • Verena Kocher $\cdot$ Luigi Mariani
}

Received: 3 February 2010/Accepted: 2 December 2010/Published online: 22 December 2010

(C) Springer Science+Business Media, LLC. 2010

\begin{abstract}
Diffusely infiltrating gliomas (WHO grade II-IV) are the most common primary brain tumours in adults. These tumours are not amenable to cure by surgery alone, so suitable biomarkers for adjuvant modalities are required to guide therapeutic decision-making. Epigenetic silencing of the $\mathrm{O}^{6}$-methylguanine-DNA methyltransferase (MGMT) gene by promoter methylation has been associated with longer survival of patients with high-grade gliomas who receive alkylating chemotherapy; and molecular testing for the methylation status of the MGMT promoter sequence is regarded as among the most relevant of such markers. We have developed a primer extension-based assay adapted to formalin-fixed paraffin-embedded tissues that enables quantitative assessment of the methylation status of the MGMT promoter. The assay is very sensitive, highly reproducible, and provides valid test results in nearly $100 \%$ of cases. Our results indicate that oligodendrogliomas, empirically known to have a relatively favourable prognosis, are also the most homogeneous entities in terms of MGMT promoter methylation. Conversely, astrocytomas, which are more prone to spontaneous progression to higher grade malignancy, are significantly more heterogeneous. In addition, we show that the degree of promoter methylation correlates with the prevalence of loss of heterozygosity on
\end{abstract}

Electronic supplementary material The online version of this article (doi:10.1007/s11060-010-0490-4) contains supplementary material, which is available to authorized users.

E. Vassella ( $₫)$ · I. Vajtai · N. Bandi · M. Arnold · V. Kocher Institute of Pathology, University of Bern, Murtenstrasse 31, 3010 Bern, Switzerland

e-mail: erik.vassella@pathology.unibe.ch

L. Mariani

Department of Neurosurgery, University Hospital of Basel,

Basel 4031, Switzerland chromosome arm 1p in the oligodendroglioma group, but not the astrocytoma group. Our results may have potentially important implications for clinical molecular diagnosis.

Keywords Oligodendroglioma $\cdot$ Astrocytoma $\cdot$ Loss of heterozygosity $1 \mathrm{p}$ and $19 \mathrm{q} \cdot$ MGMT promoter methylation . Formalin-fixed paraffin embedded tissue
Abbreviations
FFPE Formalin-fixed paraffin-embedded
LOH Loss of heterozygosity
MGMT $\mathrm{O}^{6}$-methylguanine-DNA methyltransferase
MSP Methylation-specific polymerase chain reaction
TMZ Temozolomide

\section{Introduction}

Diffusely infiltrating gliomas (WHO grade II-IV) account for some $60 \%$ of primary brain tumours in adults. Based on their microscopic morphology, the overwhelming majority of these tumours may be assigned to either the astrocytoma or the oligodendroglioma group, and a significant minority (oligoastrocytomas) are felt to involve both tumour cell types [1].

Astrocytomas of either grade (i.e., diffuse astrocytoma; anaplastic astrocytoma; glioblastoma) are notorious for pursuing a spontaneously progressive course, and tend to respond poorly to adjuvant therapeutic modalities. Oligodendroglial and oligoastrocytic tumours (i.e., oligodendroglioma; oligoastrocytoma; and anaplastic variants thereof), on the other hand, have been known to behave in a 
comparatively less aggressive manner and to be remarkably responsive to alkylating drugs (e.g., temozolomide, TMZ) [2]. They are characterized by a loss of heterozygosity (LOH) of the chromosome arms $1 p$ and 19q [3-6] which, in most cases, is caused by an unbalanced $\mathrm{t}(1 ; 19)(\mathrm{q} 10 ; \mathrm{p} 19)$ translocation $[4,7,8]$. These deletions are not only associated with longer survival of patients treated with either chemotherapy or radiotherapy or combined radiochemotherapy $[4,7,8]$, but also constitute a treatment-independent prognostic factor $[4,9,10]$.

The cytotoxic effect of TMZ is mediated by DNA alkylation of guanine residues. The DNA repair gene $\mathrm{O}^{6}$-methylguanine-DNA methyltransferase (MGMT) is responsible for removing alkyl groups from the $\mathrm{O}^{6}$ position of guanine residues. Gliomas are heterogeneous with regard to MGMT expression: in a subgroup of tumours this gene is silenced by promoter methylation (reviewed by Hegi et al. [11]). Consequently, it has been hypothesized that methylation of the MGMT promoter may be a predictor of response to alkylating chemotherapy. Indeed, Esteller et al. [12] and Hegi et al. [13, 14] showed that patients whose tumours harbour a methylated MGMT promoter sequence have significantly longer progressionfree and overall survival when treated with a combination of radiation therapy and TMZ compared with patients with an unmethylated MGMT promoter. Moreover, MGMT promoter methylation has been shown to be a treatmentindependent favourable prognostic marker that could, therefore, prospectively identify long-term survivors [14, 15]. These results were confirmed by the findings of the European Organisation for Research and Treatment of Cancer which demonstrated that MGMT promoter methylation was the strongest predictor of response to TMZ in high-grade gliomas [16]. In contrast, the role of MGMT in predicting response to TMZ in low-grade gliomas is less clearly understood.

Although MGMT promoter methylation could be routinely used as a prognostic marker in glioblastomas, there is so far no consensus on the method to be applied. In most studies, the MGMT promoter methylation status was assessed by means of methylation-specific polymerase chain reaction (MSP) followed by a nested PCR and agarose gel electrophoresis [12-14]. This method clearly correlates with clinical response, but suffers from the drawbacks that it is not quantitative and the failure rate for formalin-fixed paraffin-embedded (FFPE) tissues is relatively high. Semi-quantitative and quantitative methods including, quantitative MSP (qMSP) by real-time PCR, methylation-specific multiplex ligation-dependent probe amplification (MS-MLPA), and pyrosequencing (reviewed by Laird [17]) have also been established, and might even have higher predictive value than qualitative MSP [18]. However, qualitative MSP is the only assay to date that has been clinically validated in prospective clinical trials [19].

Here we describe a simple, primer extension-based assay which enables the methylation status of the MGMT promoter to be assessed quantitatively. This method is very sensitive, reproducible, and is adapted to FFPE tissues and thus suitable for clinical use. We compared this method with a modified MSP method for which the same primers specific for methylated and unmethylated DNA were used as for the gel-based method described by Esteller et al. [12] and Hegi et al. [13, 14], and demonstrate that both methods are in good agreement. To our knowledge, no study has been reported on the heterogeneity of the intratumoural methylation pattern of the MGMT promoter among glioma entities. We provide evidence that glioblastomas, as a group, have a significantly more heterogeneous pattern of promoter methylation of the MGMT gene than oligodendrogliomas; this may potentially have implications for the patient's response to alkylating agents.

\section{Materials and methods}

Patient samples and cell lines

A total of 31 patients with oligodendrogliomas (WHO grade II-III; mean age: 54 years; 17 male/14 female), 40 patients with oligoastrocytomas (WHO grade II-III; mean age: 47 years; 24 male/16 female), 55 patients with astrocytomas (WHO grade II-III; mean age: 44 years; 30 male/ 25 female), and 164 patients with glioblastoma (WHO grade IV; mean age: 59 years; 104 male/60 female) were analysed. Tissue diagnosis and specimen selection were performed in a specialized surgical neuropathology setting by two neuropathologists (MA and IV). Tumour classification and grading was performed according to the criteria defined by the current WHO Classification of Tumours of the Central Nervous System [1]. All experiments using human specimens were conduct in compliance with the ethical guidelines of the Institute of Pathology at the University of Bern and were reviewed by the institutional review board.

The human glioblastoma cell line U87 was kindly provided by M.E. Hegi, University of Lausanne.

\section{DNA extraction and DNA modification}

The age range of the FFPE tissue blocks used for DNA extraction was between 1989 and 2008; 16\% of the paraffin blocks were older than four years. Tissue blocks were carefully inspected by a neuropathologist. Tumour areas of the infiltration zone, large necrotic regions, or regions containing large numbers of inflammatory cells or blood 
vessels were excluded. Representative areas of tumour containing at least $70 \%$ neoplastic cells were excised from FFPE tissues and DNA was extracted using the EZ1 tissue kit and the BioRobot EZ1 workstation (Qiagen, Hombrechtikon, Switzerland) according to the manufacturer's procedure with the modification that tissues were heattreated in lysis buffer for $10 \mathrm{~min}$ at $95^{\circ} \mathrm{C}$ before proteinase $\mathrm{K}$ digestion. For $\mathrm{LOH}$ analysis, reference DNA was extracted from the patient's EDTA peripheral blood using the EZ1 tissue kit. The same kit was also used for extraction of DNA from cell lines.

Bisulfite treatment was performed using the EZ DNA modification kit according to the manufacturer's procedure (Zymo, Orange, CA, USA). The modified DNA was eluted with $10 \mu \mathrm{l}$ M-elution buffer and diluted with $10 \mu \mathrm{l}$ water. A control containing a mixture of equal amounts of methylated (U87) and unmethylated (PBMC) DNA and a no-DNA control were included in each experiment.

\section{Quantitative MSP}

In a first step, bisulfite-treated DNA was amplified by PCR using the primer pair MU_forward (5'-GGATATGTT GGGATAGTT- $3^{\prime}$ ) and MU_rv (5'-ACGCCTACAAAAC CACTC- $3^{\prime}$ ) and $2 \mu \mathrm{l}$ bisulfite-treated DNA. Both primer binding sites contain no $\mathrm{CpG}$ dinucleotides, however, they encompass $\mathrm{CpG}$ dinucleotides that are relevant for predicting chemoresistance. DNA from FFPE tissues is highly fragmented. Therefore, primers were designed giving rise to a short amplicon of only $169 \mathrm{bp}$ which enables efficient amplification of fragmented DNA.

In a second step, the PCR product was diluted 1:10 with water and $2 \mu \mathrm{l}$ was subjected to a multiplex primer extension in a reaction containing $0.5 \mu \mathrm{M}$ primer $\mathrm{M}_{-}$forward_HEX (5'-GCACTCTTCCGAAAACGAAACG-3'), $0.5 \mu \mathrm{M}$ primer U_forward_FAM (5'-AACTCCACACTCT TCCAAAAACAAAACA-3' $\left.{ }^{\prime}\right), 800 \mu \mathrm{M}$ dNTPs, $1 \mathrm{U}$ Taq, and $1 \times$ Taq buffer (Applied Biosystems) in a total volume of $30 \mu$ l. The forward primers were tagged with hexachlorofluorescein (HEX) or 6-carboxyfluorescein (FAM), respectively (Microsynth, Balgach, Switzerland). The sequences of the primers are the same as those reported previously $[12-14,20]$. Primer extension was performed for five cycles in a GeneAmp 9700 thermal cycler (Applied Biosystems) using the conditions: $94^{\circ} \mathrm{C}$ for $30 \mathrm{~s}, 62^{\circ} \mathrm{C}$ for $30 \mathrm{~s}$, and $72^{\circ} \mathrm{C}$ for $30 \mathrm{~s}$. The product was diluted 1:5 with water and analysed by capillary electrophoresis using a genetic analyser (ABI Prism 3100-Avant; Applied Biosystems, Rotkreuz, Switzerland) and GeneMapper software 4.0.

The percentage of methylated DNA was determined by measuring the peak areas for methylated and unmethylated DNA. The relative amount of methylated DNA was normalized to the ratio of signal intensities obtained for a control containing equal amounts of methylated and unmethylated DNA performed in the same experiment. DNA from peripheral blood monocytes was included as a negative control, which consistently gave rise to completely unmethlylated DNA.

\section{Qualitative MSP}

Qualitative MSP was performed using primer pairs specific for methylated (M_forward_HEX and M_rv (5'-GTTTCG ACGTTCGTAGGTTTTCGC-3') ) or unmethyled (U_forward_FAM and U_rv (5'-GTTTGTGTTTTGATGTTTG TAGGTTTTTGT- $\left.3^{\prime}\right)$ ) DNA of the MGMT promoter and $2 \mu$ bisulfite-treated DNA, which amplify fragments of 122 and 129-bp, respectively. In contrast with the published MSP method [12-14], the first PCR step of the nested PCR procedure was omitted. Alternatively, amplification was performed using the primer pair MU_forward and MU_rv and the diluted product was re-amplified using either the primer pair M-forward_HEX and M_rv or the primer pair U_forward_FAM and U_rv. Analysis of PCR products was performed by capillary electrophoresis.

\section{$\mathrm{LOH}$ analysis}

LOH analysis was performed exactly as described elsewhere [10] using fluorescent primers tagged with HEX or FAM for microsatellite markers on chromosome 1p36 (D1S468, D1S1612, D1S228 and D1S214) and chromosome 19q13 (D19S219, D19S412 and D19-HRC). PCR products were analysed by capillary electrophoresis. Diagnostic criteria for $\mathrm{LOH}$ required the calculated ratio of the peak areas to be less than 0.5 .

\section{Cloning and sequencing}

Bisulfite-treated DNA was amplified by PCR using the primer pair MU_forward and MU_rv and cloned into pGEM-T easy vector (Promega, Madison, WI, USA) according the manufacturer's procedure. The methylation status of the MGMT promoter was determined by sequencing of ten individual clones.

\section{Statistics}

Statistical analysis was performed by use of GraphPAD prism software (San Diego, CA, USA). Statistical differences were calculated using an unpaired two-tailed Student's $t$-test. A probability of $P<0.05$ was considered statistically significant. Statistical significance of correlation was calculated by use of the $N-1 \chi^{2}$ test $(2 \times 2$ table Pearson's analysis). 


\section{Results}

Establishment of a primer extension-based quantitative MGMT promoter methylation assay

The successive reaction steps for the quantitative MGMT promoter methylation assay are shown schematically in Fig. 1a. In a first step, bisulfite-treated genomic DNA was amplified using primers that are equally able to bind methylated and unmethylated DNA. Both primer sites encompass diagnostically relevant $\mathrm{CpG}$ dinucleotides [12-14, 20]. To assess the methylation status, a multiplex primer extension was performed using fluorescent-labelled primers, which bind to $\mathrm{CpG}$ dinucleotides specific for the methylated or unmethylated form of the DNA, followed by capillary electrophoresis. If the assay were truly quantitative, we would expect that the relative signal intensity of fluorescent products reflects the proportion of methylated DNA.

The quantitative assay was compared with a modified version of the commonly used qualitative, gel-based assay. In this case, bisulfite-treated DNA was amplified using

A

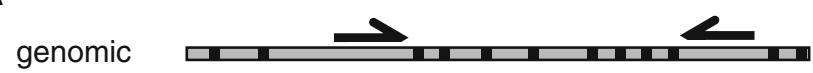
DNA
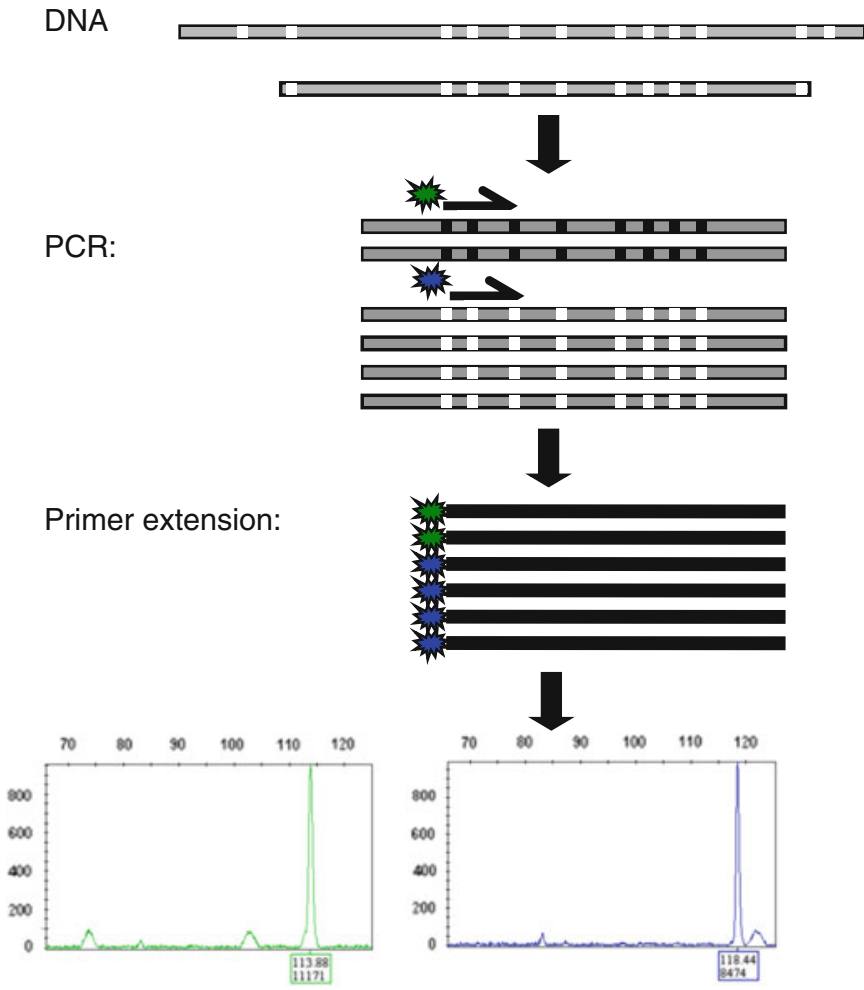

Fig. 1 Schematic depiction of reaction steps used for the quantitative (a) and qualitative (b) analysis of MGMT promoter methylation. Grey boxes indicate genomic DNA or PCR products, small black boxes fluorescent-labelled primers specific for the methylated or unmethylated form of the DNA, and analysed by capillary electrophoresis (Fig. 1b).

Assay validation

Different control experiments were performed to validate the quantitative methylation assay. To investigate the specificity of the assay, untreated genomic DNA was analysed; this, however, did not yield any product (data not shown). Likewise, the primer specific for the unmethylated form was unable to bind to bisulfite-treated, methylated DNA (extracted from U87 cells), and the primer specific for the methylated form was unable to bind to bisulfitetreated, unmethylated DNA (extracted from peripheral blood monocytes, data not shown).

To investigate whether the assay was quantitative, genomic DNA from U87 and PBMC cells was mixed at different concentrations and analysed for methylation status. In Fig. 2, the relative intensity of the fluorescent signal for methylated and unmethylated DNA is plotted against real the proportions of both DNAs. The relative fluorescence

\section{B}
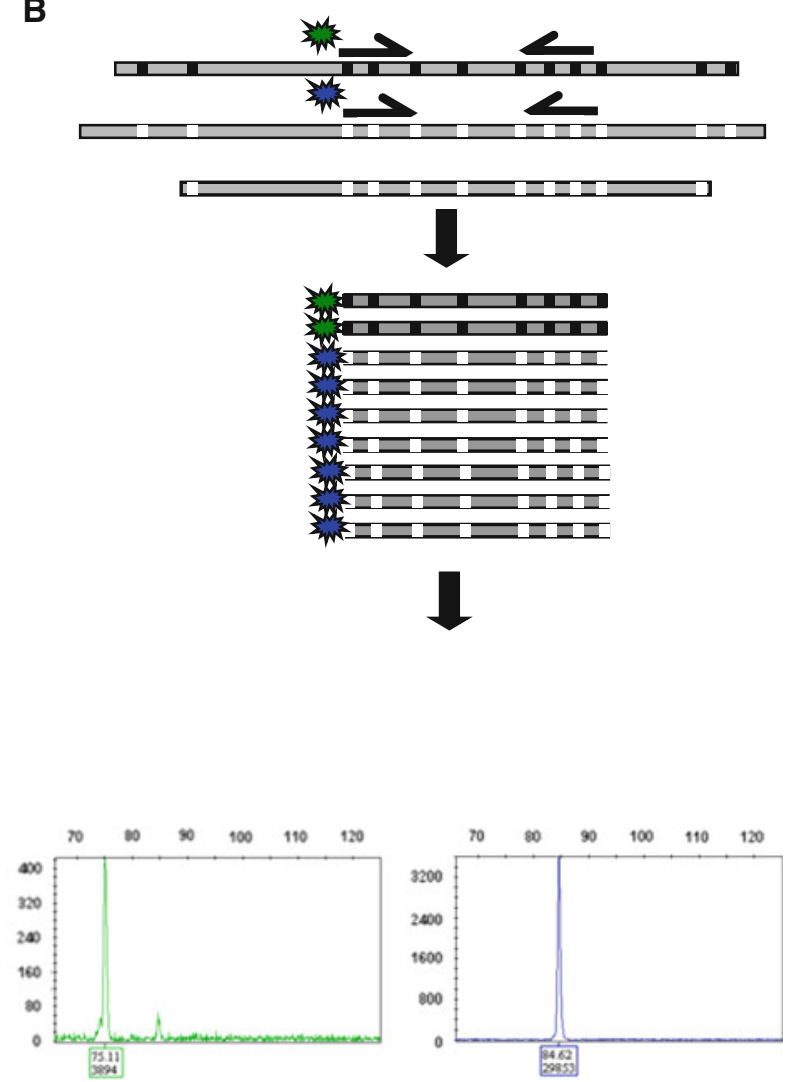

indicate methylated $\mathrm{CpG}$ islands and open boxes indicate unmethylated $\mathrm{CpG}$ islands (not drawn to scale). Arrows indicate primers used for PCR and primer extension 
intensity of the primer extension products clearly reflects the proportion of methylated DNA (Fig. 2; $r=0.977$ ).

Tissue samples in pathology departments are routinely stored as formalin-fixed and paraffin-embedded (FFPE) tissue blocks. This preparation is unsuitable for many downstream molecular biology techniques, because of extensive fragmentation and modification of nucleic acids. As a consequence, the commonly used gel-based MGMT methylation assay, which gives rise to a PCR product of $290 \mathrm{bp}$, failed to yield amplification products in $25-33 \%$ of cases [14, 21]. In contrast, both the qualitative and the quantitative method used in this study, which give rise to significantly shorter amplicons $(<170 \mathrm{bp})$ than the gelbased method, provided amplification products for all 290 glial tumours that were analysed. Sixteen percent of the paraffin blocks were older than four years and some were up to 20 years old; all of these provided informative results.

To investigate whether the quantitative assay is reproducible using FFPE material, a series of ten glioma cases, nine of which were positive by the qualitative assay, were analysed using the quantitative assay. Figure 3 a illustrates the reproducibility among independent replicates, in particular at higher values (SD maximum $\pm 20 \%$ ). In conclusion, the quantitative assay is suitable for analysis of FFPE material.

Finally, the sensitivity of the assay was determined by DNA titration experiments. As shown in Fig. 3b, MGMT promoter methylation could be determined from as little as $0.4 \mathrm{ng}$ genomic DNA.

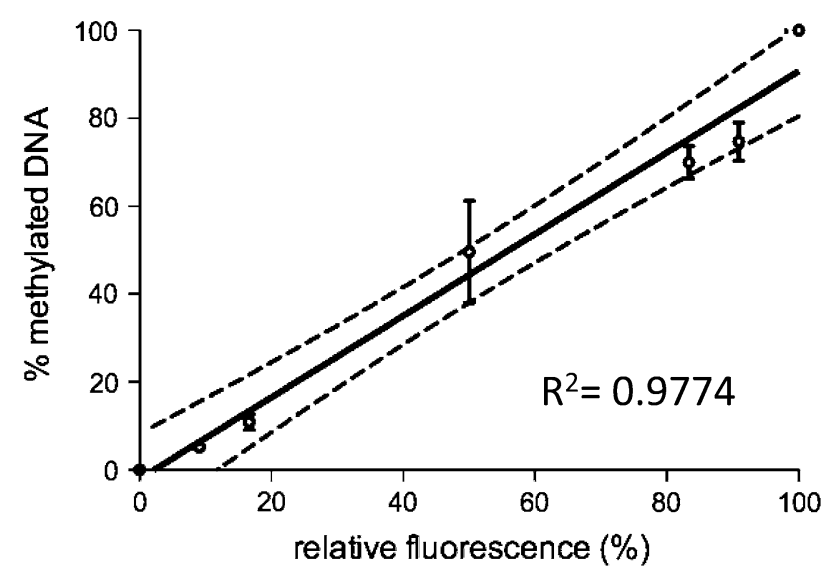

Fig. 2 Linear correlation between the percentage of methylated DNA and relative fluorescence signal. Genomic DNA from U87 (100\% methylated) and PBMC cells (unmethylated) were mixed at different concentrations and analysed using the quantitative assay. The relative intensity of the fluorescence signal for methylated and unmethylated DNA is plotted against the real proportions of methylated DNA $(n=3)$
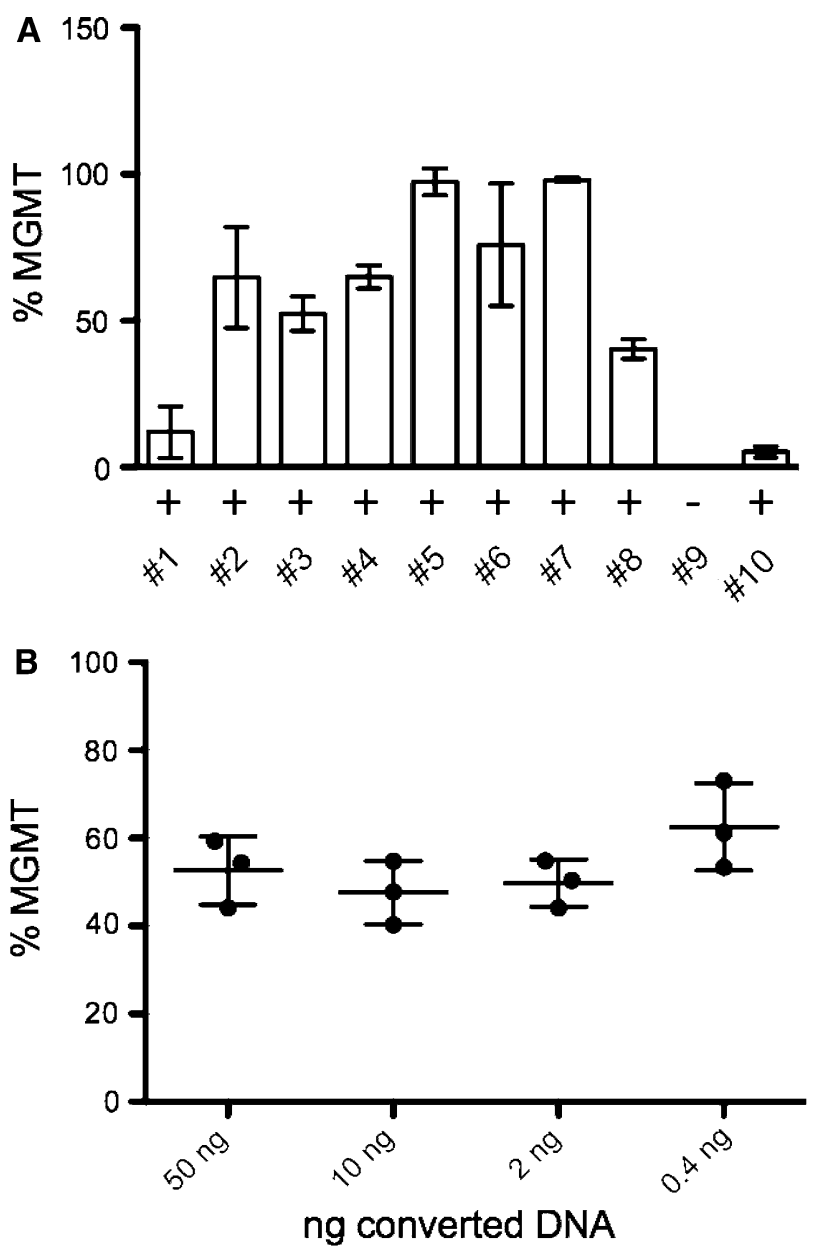

Fig. 3 Reliability of the quantitative assay. a Reproducibility. Glioma tissues were excised from FFPE material and the percentage of methylated DNA for the MGMT promoter was determined using the quantitative assay. Values were normalized to a control containing equal amounts of methylated and unmethylated DNA and presented as mean $\pm \mathrm{SD}(n=4)$ relative to methylated DNA. Replicates are derived from independent DNA conversion experiments. The result of the qualitative analysis is shown below. b Sensitivity. Bisulfite-treated DNA containing equal amounts of methylated and unmethylated DNA was titrated and analysed using the quantitative method. Values are presented as mean $\pm \mathrm{SD}(n=3)$

Comparison of qualitative and quantitative methods

As indicated in Fig. 3a, analysis of a small number of tumours revealed that the quantitative and qualitative assays are in very good agreement (Fig. 3a). To address this issue on a larger scale, 290 glial tumours were compared for MGMT promoter methylation using the qualitative and quantitative assays. Samples with detectable methylation were scored as positive. As shown in Table 1, concordant test results were obtained for $\sim 90 \%$ of samples $(P<0.0001)$. Among the 30 samples that provided discordant results, 29 were MGMT methylation-positive by 
Table 1 Correlation of quantitative and qualitative assays for MGMT promoter methylation

\begin{tabular}{|c|c|c|c|c|}
\hline \multirow[t]{2}{*}{ Qualitative PCR } & \multicolumn{2}{|c|}{ Quantitative $\mathrm{PCR}^{\mathrm{a}}$} & \multirow[t]{2}{*}{$n$} & \multirow[t]{2}{*}{$P^{\mathrm{b}}$} \\
\hline & - & + & & \\
\hline MGMT+ & 1 & 128 & 290 & $<0.0001$ \\
\hline MGMT- & 132 & 29 & & \\
\hline
\end{tabular}

a Samples with \% MGMT $>0$ were scored as positive

b $P$-values were calculated by the $\chi^{2}$ method (two by two table Pearson's analysis, two-tailed)

the quantitative assay and only one was methylationnegative. In most of these cases, the quantitative assay gave rise to a relatively low fluorescence signal for the methylated form of DNA, suggesting that this method is more sensitive than the qualitative assay. Consistent with these findings, 25 out of 28 samples turned out to be MGMTpositive when subjected to PCR using the primer pair MU_forward and MU_rv followed by a nested PCR using the methylation-specific primers (data not shown). However, for some samples, discordant results were reproducibly obtained although the fluorescent signal for the methylated form was relatively high by the quantitative assay.

To better understand the discrepancy of results obtained by the two methods, two samples were selected for further analysis. For both samples approximately $80 \%$ MGMT promoter methylation was observed, as indicated by the quantitative assay, but only sample \#2 was positive by the qualitative assay. Bisulfite-treated DNA was amplified using the primer pair MU_forward and MU_rv and cloned into pGEM-T easy vector. Ten individual clones were selected from each sample and sequenced. The result of this analysis is summarized in Fig. 4. Cytosine residues that do not form part of $\mathrm{CpG}$ dinucleotides were all converted to thymidine indicating that bisulfite conversion was efficient. Interestingly, both samples revealed a relatively high degree of methylation of $\mathrm{CpG}$ dinucleotides, despite the fact that sample \#1 was negative by the qualitative assay. Both samples showed a heterogeneous methylation pattern within the binding site of primer M_forward (Fig. 4, upper grey box) suggesting that binding of this primer to its target DNA requires the formation of mismatches in order that the polymerase reaction can proceed. However, the finding that the quantitative, but not the qualitative, assay provided a positive result for sample \#1, indicates that the latter assay is more restrictive for fully methylated DNA. Consistent with this result, the degree of methylation within the binding site of primer M_forward was higher for sample \#2 than for sample \#1.

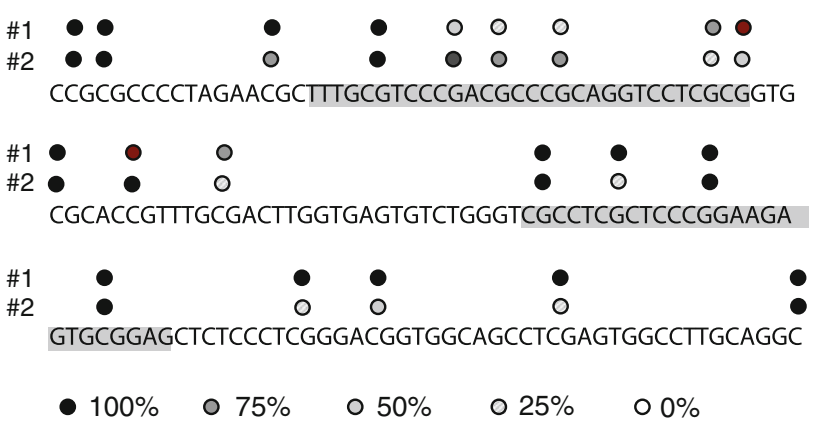

Fig. 4 Frequency of methylation of individual $\mathrm{CpG}$ islands. PCR products of the quantitative assay were subcloned and ten independent clones were sequenced. The percentage of methylated DNA, as indicated by the number of clones containing a cytosine residue at a specific position, was determined. Both samples gave rise to $80 \%$ methylation using the quantitative assay, but only sample \#2 provided a positive result by the qualitative assay. Primer sites used for primer extension (quantitative method; upper grey box) and primer sites for PCR (qualitative method; upper and lower grey box) are indicated

Gliomas are heterogeneous for MGMT promoter methylation

It may be inferred from the results in Fig. 3a that individual glioma cells within a given tumour sample are remarkably inhomogeneous with regard to MGMT promoter methylation. One might object that "intrinsic contamination" of tumour tissue samples by non-neoplastic intratumoral accessory structures (e.g., blood vessels, hematogeneous inflammatory cells, microglia, etc.) might contribute to such heterogeneity. However, care was taken to select tumour tissues containing at least $70 \%$ of neoplastic tissues. To determine the relative amount of tumour DNA in our samples, we took advantage of the fact that a relatively high proportion of gliomas exhibit deletion of chromosome arms $1 \mathrm{p}$ and/or 19q. LOH was assessed for microsatellite markers on chromosomal regions $1 \mathrm{p} 36$ and $19 \mathrm{q} 13$. The allelic status of tumour DNA was compared with the DNA from the patient's peripheral blood. If a tumour with a LOH $1 p / 19 q$ consists of pure tumour cells we would expect a complete loss of one allelic site of an informative microsatellite marker. In contrast, contamination with stroma cells leads to an incomplete loss of this region (Electronic Supplementary Material, Fig. 1). The relative loss of signal intensity of a microsatellite in the tumour tissue plotted against the signal intensity of that same microsatellite marker in the normal tissue can be used as a measure of the proportion of tumour cells in the preparation [22]. In our series, 21 oligodendrogliomas, 24 oligoastrocytomas, 9 astrocytomas and 52 glioblastomas contained an LOH 1p and/or 19q. In Fig. 5, the percentage of methylated DNA of tumour samples corrected for the relative signal intensity of microsatellites on chromosomes 


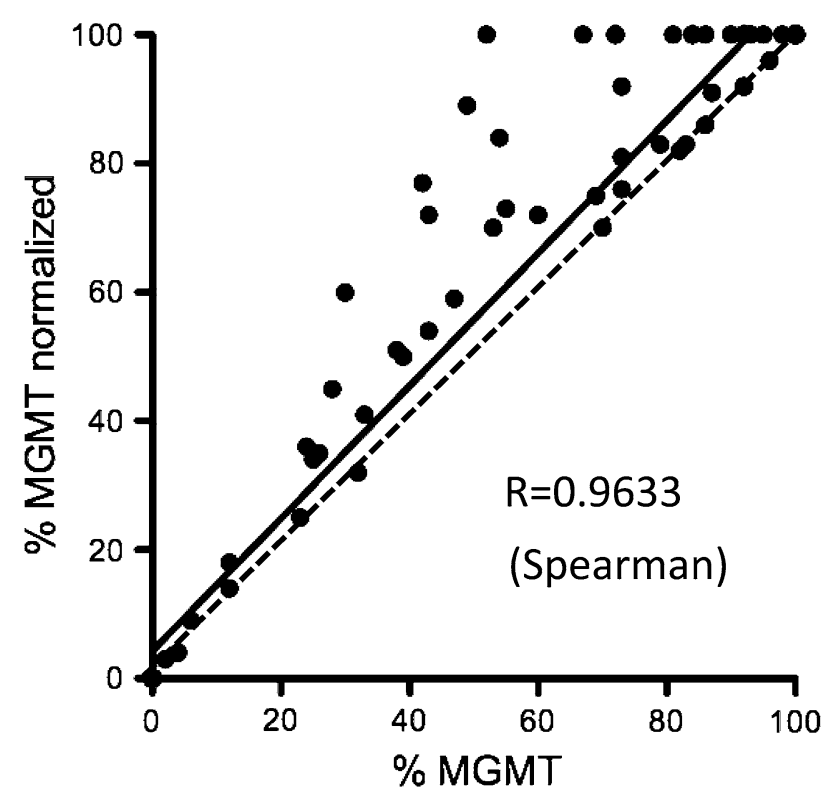

Fig. 5 Percentage of methylated DNA in the tumour samples corrected for the relative amount of tumour DNA. The relative amount of tumour DNA in the tissue samples was calculated from the loss of signal intensity of informative microsatellites on chromosome arms $1 \mathrm{p}$ or $19 \mathrm{q}$ in the tumour sample relative to a reference DNA from peripheral blood monocytes from the same patient. The percentage of methylated DNA corrected for the relative amount of tumour DNA was plotted against uncorrected values. Data points for tissue samples containing $100 \%$ tumour DNA align along the diagonal dot line $(x=y)$ in the dot blot. Only tumours which contain an LOH $1 \mathrm{p}$ and/or $19 \mathrm{q}$ were included. For direct comparison, LOH and MGMT status were assessed from the same DNA sample. The solid line represents the linear regression. Spearman correlation 0.96 , $n=74$

$1 \mathrm{p}$ or $19 \mathrm{q}$ was plotted against uncorrected values. Data points for tissue samples containing $100 \%$ tumour cells align along the diagonal dot line in the dot blot (Fig. 5). Figure 5 illustrates that most data points fall on or near the diagonal line indicating that most samples were not significantly contaminated with non-neoplastic cells. From these results we may conclude unequivocally that gliomas have relatively high heterogeneity in the pattern of promoter methylation of individual neoplastic cells.

\section{Comparison of glioma subtypes for MGMT} promoter methylation

In agreement with published results [11], the quantitative MGMT promoter methylation assay provided at least some degree of promoter methylation in $81 \%(25 / 31)$ of oligodendrogliomas (WHO grade II-III), 68\% (27/40) of oligoastrocytomas (WHO grade II-III), 42\% (23/55) of astrocytomas (WHO grade II-III), and 50\% (82/164) of glioblastomas (WHO grade IV). However, it is unknown if glioma entities also differ in their relative degree of

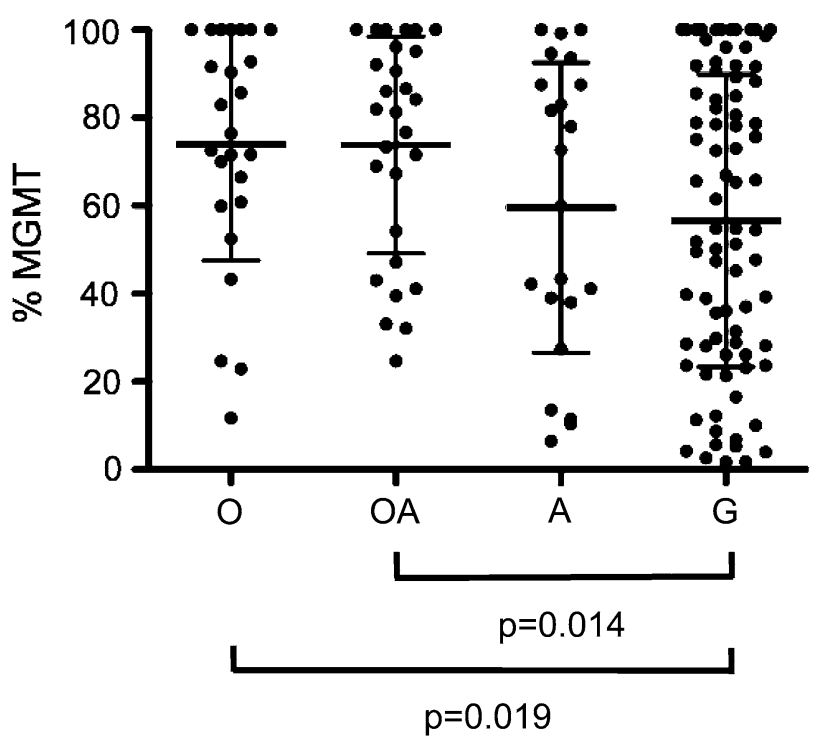

Fig. 6 Comparison of the degree of promoter methylation between oligodendrogliomas $(O)$, oligoastrocytomas $(O A)$, astrocytomas $(A)$, and glioblastomas $(G)$. Statistical differences were calculated using unpaired two-tailed $t$-test

intratumoural methylation heterogeneity. As shown in Fig. 6, methylation-positive oligodendrogliomas and oligoastrocytomas turned out to be the most homogeneous groups with an average percentage of cells with promoter hypermethylation of $75 \%$. An increased tendency for intratumoural methylation heterogeneity was noted for methylation-positive astrocytomas (59\%), but this did not reach statistical significance. In contrast, methylationpositive glioblastomas were highly heterogeneous for MGMT promoter methylation, which was statistically significant, with an average percentage of cells with hypermethylated MGMT promoter of 55\% (Fig. 6).

Correlation between MGMT promoter methylation and $\mathrm{LOH} 1 \mathrm{p}$

As shown in Fig. 7a, there was also a significant difference in the percentage of cells with promoter hypermethylation of oligodendrogliomas and oligoastrocytomas with $\mathrm{LOH}$ 1p (69\%), compared with tumours without LOH (30\%) $(P<0.0001)$. In contrast, no significant correlation was noted between the incidence of promoter methylation, as indicated by the qualitative assay, and the incidence of LOH $1 \mathrm{p}$.

Within the astrocytoma group (including glioblastomas) no correlation was observed between the degree of promoter methylation and incidence of $\mathrm{LOH} 1 \mathrm{p}$ (Fig. 7b) irrespective of the method used for MGMT analysis. Notably, the degree of promoter methylation for both subgroups (24-32\%), as indicated by the quantitative 

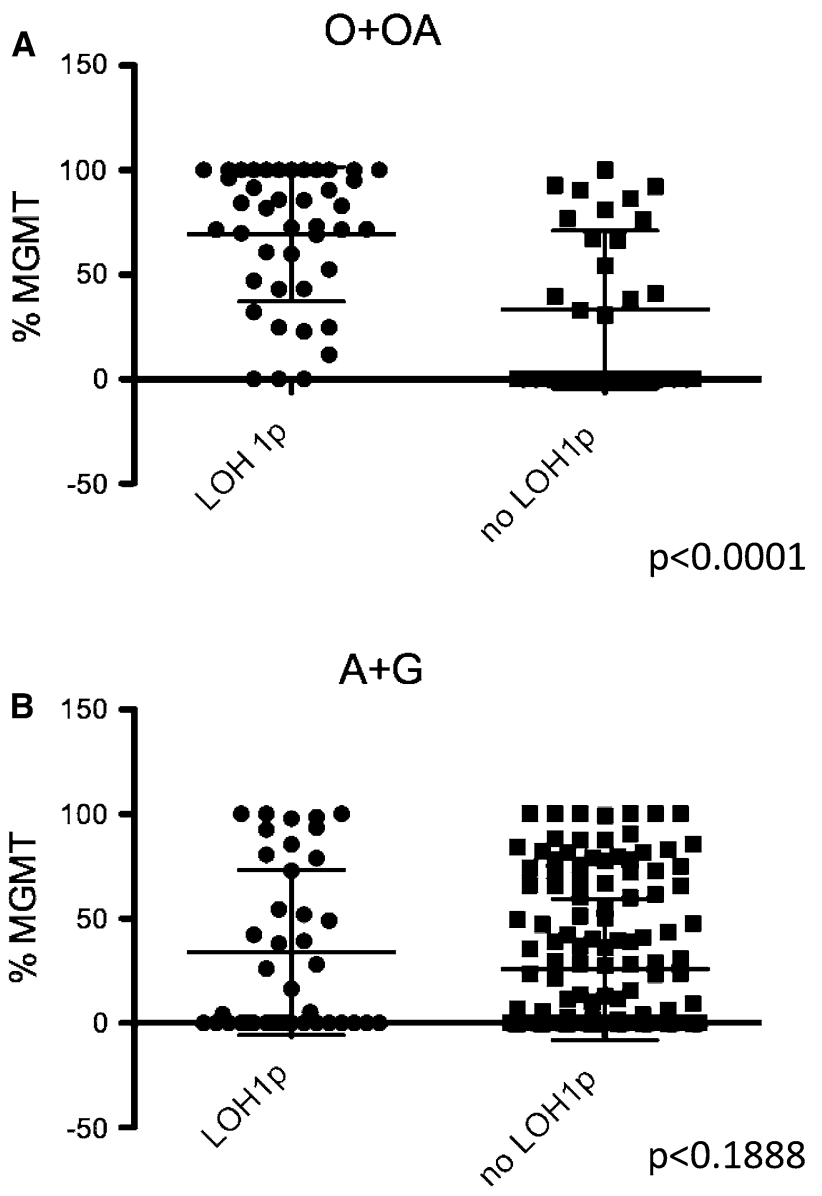

Fig. 7 Degree of promoter methylation of gliomas with LOH $1 p$ compared with gliomas without LOH 1p. a Oligodendrogliomas and astrocytomas. b Astrocytomas and glioblastomas. Statistical differences were calculated using unpaired two-tailed $t$-test

method, was similar to that obtained for oligodendrogliomas and oligoastrocytomas without LOH 1p.

\section{Discussion}

Over the past few years, a series of different methods, including methylation-specific PCR [12, 14, 20, 23], combined bisulfite restriction analysis [24], qMSP [21, 25], single-nucleotide primer extension [26], MS-MLPA [27], and pyrosequencing [28], have been established for determining promoter methylation of the MGMT gene, but it remains unclear which assay performs best in predicting TMZ sensitivity in gliomas. Some of these methods may not be suitable for clinical settings, for they are either too complex, prone to false-positive or false-negative results (in particular if nested PCR is used), or provide no valid result in a high proportion of cases. Quantitative methods intuitively prove more reliable than qualitative assays, which may be because a heterogeneous methylation pattern of the MGMT promoter is very frequent in high-grade gliomas. In addition, low degrees of MGMT methylation can also be detectable in normal tissues including brain tissue of some elderly individuals [29-31], which may lead to false-positive results when qualitative methods are applied. Analysis of MGMT protein expression by immunohistochemistry will probably not gain wide acceptance, because it is clearly less reliable and performs poorly when compared with actual MGMT promoter methylation status [32].

Here, we contribute an additional, primer extensionbased method, which enables quantitative assessment of the methylation status of the MGMT promoter. This method maps six diagnostically relevant $\mathrm{CpG}$ dinucleotides [12-14, 20] including CpG4, which yielded the best prediction among the $\mathrm{CpG}$ dinucleotides within this island for overall survival in patients initially treated with radiotherapy and TMZ [18].

This method is sensitive and consistently reproducible using routinely processed histopathology samples (i.e., FFPE). In addition, it provides valid test results in a very high percentage of cases. Although there is generally good agreement between this method and the qualitative assay used in this study, we feel that the quantitative method may particularly appeal because of its robustness. Because not every $\mathrm{CpG}$ site is methylated in methylation-positive tumours, PCR-based methods have in common that binding of the primer specific for methylated DNA to its target DNA requires the formation of mismatches, which may result in a lower efficiency of the polymerase reaction. However, this has a much greater effect on PCR amplification, for which high numbers of cycles are used (for example in the case of MSP or qMSP), compared with primer extension, for which only a few cycles are used. In addition, MSP or qMSP normally interrogates more $\mathrm{CpG}$ sites, because these methods depend on two methylationspecific primers for the amplification and thus require more sites to be methylated compared with primer extensionbased PCR. However, higher selectivity for fully methylated DNA is at the expense of lower sensitivity.

The qMSP assay yields binary data without a quantitative measure of the contribution from unmethylated DNA. Consequently, tumours with high proportions of tumour cells with hypermethylated MGMT promoter will score the same in the qMSP assay as tumours bearing only a few hypermethylated cells, thus underestimating the heterogeneity of methylation. Moreover, qMSP may result in underestimation of methylation in a tumour with gain of chromosome seven if ACTB (which is located on this chromosome) is used for normalisation [33]. In contrast, pyrosequencing and primer-extension based PCR may be a better estimate of the degree of intratumoral heterogeneity of MGMT methylation. However, neither of these methods 
enables us to discriminate whether the heterogeneity of the methylation pattern is because of the presence of two populations of tumour cells, one with a high degree and one with a low degree of promoter methylation, or if this is because of patchiness within a particular DNA strand. However, on the basis of our results we can exclude the possibility that the observed heterogeneity is because of contamination with non-neoplastic cells.

On the basis of pyrosequencing [18, 34] or bisulfite sequencing [35, 36], several studies have claimed methylation heterogeneity of the MGMT promoter in glioblastomas, although this has been challenged by others [37]. In contrast, oligodendrogliomas seem to be more homogeneous [28], but no study exists in which both tumour entities were directly compared. It may also be questioned in some cases to what extent non-neoplastic cells may contribute to this heterogeneity. By analysing 290 diffusely infiltrating gliomas of adults with primerextension based PCR, we were able to confirm that glioblastomas are indeed highly heterogeneous with regard to promoter methylation of the MGMT gene while oligodendrogliomas and oligoastrocytomas are significantly more homogeneous. It is conceivable that patients may respond less well to chemotherapy if the tumour is heterogeneous for MGMT methylation, because this is likely to result in rapid selection of tumour cells with a low methylation status in the microevolutionary context of a given glioma. In line with this notion, we show by our quantitative method that the degree of promoter methylation correlates with overall survival in a small cohort of 60 glioblastoma patients treated with TMZ (Electronic Supplementary Material, Fig. 2a). Notably, patients with $>70 \%$ methylation of their tumour DNA had the longest overall survival (28 months), compared with 10 months for patients with unmethylated promoter $(P=0.021)$. In contrast, patients with a degree of methylation $<70 \%$ had an life expectancy (17 months) intermediate between those with unmethylated promoter and those with hypermethylated promoter. Very consistent results were also obtained by Dunn et al. [34] in a cohort of 109 glioblastoma patients which were analysed by pyrosequencing. In addition, in a retrospective study of low-grade gliomas, we showed that the volumetric response to chemotherapy correlates with the level of MGMT-promoter methylation [38]. Single step or nested MSP gave rise to a mean overall survival of 10-12 months for patients with unmethylated MGMT promoter and 24-28 months for patients with methylated MGMT promoter (Electronic Supplementary Material, Figs. 2b, c). In conclusion, all methods enable prediction of survival, but the quantitative method enables further prediction of the magnitude of response to TMZ. It remains to be shown whether oligodendrogliomas are more sensitive to TMZ than astrocytomas, solely by virtue of their more homogeneous MGMT promoter methylation status.

LOH $1 p$ and $19 q$ is regarded as an additional marker for response to chemotherapy and, like MGMT promoter methylation, is frequently detected in oligodendrogliomas. Thus, an important question is whether these two genotypic features are causally linked in oligodendrogliomas. Interestingly, MGMT promoter methylation was prognostic and not predictive for the outcome to adjuvant chemotherapy in anaplastic oligodendroglial tumours [39, 40] and correlated with other prognostic factors, for example $1 \mathrm{p} 19 \mathrm{q}$ co-deletions and isocitrate dehydrogene gene mutations [2, 28]), in particular if quantitative methods for MGMT methylation were used. Consistent with these results, a significant correlation was noted in our study when the MGMT promoter methylation status was assessed quantitatively. However, no such correlation was seen in the astrocytoma group, which has not been addressed so far. These results may suggest that in oligodendrogliomas these markers may reflect the less aggressive nature of these tumours, but the underlying molecular mechanisms are unknown. In the astrocytoma group, however, 1p19q deletions and promoter methylation seem to be unlinked. It is possible that these tumours may carry a general defect in regulating DNA methylation leading to epigenetic silencing of multiple genes including those linked to chemoresistance.

Our results indicate that quantitative analysis of the methylation status of the MGMT promoter may be resorted to for predicting the likelihood of response of glioma patients to chemotherapy. Quantitative assessment of the MGMT promoter methylation status, stratification of clinical response to chemotherapy, and prognosis must be correlated in a larger future study.

Acknowledgments This research was supported by a grant from the Swiss National Science Foundation and the Bernese Cancer League.

\section{References}

1. Louis D, Ohgaki H, Wiestler O, Cavenee W, Burger P, Jouvet A, Scheithauer B, Kleihues P (2007) The 2007 WHO classification of tumours of the central nervous system. Acta Neuropathol 114:97-109

2. van den Bent MJ, Hegi ME, Stupp R (2006) Recent developments in the use of chemotherapy in brain tumours. Eur $\mathrm{J}$ Cancer 42:582-588

3. Reifenberger G, Louis DN (2003) Oligodendroglioma: toward molecular definitions in diagnostic neuro-oncology. J Neuropathol Exp Neurol 62:111-126

4. Smith JS, Perry A, Borell TJ, Lee H, O'Fallon J, Hosek S, Kimmel D, Yates A, Burger P, Scheithauer B, Jenkins R (2000) Alterations of chromosome arms $1 \mathrm{p}$ and $19 \mathrm{q}$ as predictors of survival in oligodendrogliomas, astrocytomas and mixed oligoastrocytomas. J Clin Oncol 18:636-645 
5. Thiessen B, Maguire J, McNeil K, Huntsman D, Martin MA, Horsman D (2003) Loss of heterozygosity for loci on chromosome arms $1 \mathrm{p}$ and $19 \mathrm{q}$ in oligodendroglioma tumours: relationship to outcome and chemosensitivity. J Neurooncol 64:271-278

6. Kujas M, Lejeune J, Benouaich-Amiel A, Crinière E, LaigleDonadey F, Marie Y, Mokhtari K, Polivka M, Bernier M, Chretien F, Couvelard A, Capelle L, Duffau H, Cornu P, Broët P, Thillet J, Carpentier A, Sanson M, Hoang-Xuan K, Delattre J (2005) Chromosome 1p loss: a favorable prognostic factor in low-grade gliomas. Ann Neurol 58:322-326

7. Jenkins R, Blair H, Ballman KV, Giannini C, Arusell R, Law M, Flynn H, Passe S, Felten S, Brown P, Shaw E, Buckner J (2006) A $\mathrm{t}(1 ; 19)(\mathrm{q} 10 ; \mathrm{p} 10)$ translocation mediates the combined deletions of $1 \mathrm{p}$ and $19 \mathrm{q}$ and predicts better prognosis of patients with oligodendroglioma. Cancer Res 66:9852-9861

8. Griffin C, Burger P, Morsberger L, Yonescu R, Swierczyknski S, Weingart JD, Murphy K (2006) Identification of der $(1 ; 19)(\mathrm{q} 10 ; \mathrm{p} 10)$ in five oligodendrogliomas suggests a mechanism of concurrent $1 p$ and $19 q$ loss. J Neuropathol Exp Neurol 65:988-994

9. Hoang-Xuan K, Capelle L, Kujas M, Taillibert S, Duffau H, Lejeune J, Polivka M, Crinière E, Marie Y, Mokhtari K, Carpentier A, Laigle F, Simon J, Cornu P, Broët P, Sanson M, Delattre J (2004) Temozolomide as initial treatment for adults with low-grade oligodendrogliomas or oligoastrocytomas and correlation with chromosome $1 \mathrm{p}$ deletions. J Clin Oncol 22:3133-3138

10. Mariani L, Deiana G, Vassella E, Fathi AR, Murtin C, Arnold M, Vajtai I, Weis J, Siegenthaler P, Schobesberger M, Reinert M (2006) Loss of heterozygosity $1 \mathrm{p} 36$ and $19 \mathrm{q} 13$ is a prognostic factor for overall survival in patients with diffuse WHO grade 2 gliomas treated without chemotherapy. J Clin Oncol 24:4758-4763

11. Hegi ME, Murat A, Lambiv WL, Stupp R (2006) Brain tumors: molecular biology and targeted therapies. Ann Oncol 17:191-197

12. Esteller M, Garcia-Foncillas J, Andion E, Goodman SN, Hidalgo OF, Vanaclocha V, Baylin SB, Herman JG (2000) Inactivation of the DNA-repair gene MGMT and the clinical response of gliomas to alkylating agents. N Engl J Med 343:1350-1354

13. Hegi ME, Diserens AC, Godard S, Dietrich PY, Regli L, Ostermann S, Otten P, Van Melle G, de Tribolet N, Stupp R (2004) Clinical trial substantiates the predictive value of O-6-methylguanine-DNA methyltransferase promoter methylation in glioblastoma patients treated with temozolomide. Clin Cancer Res 10:1871-1874

14. Hegi ME, Diserens A-C, Gorlia T, Hamou M, de Tribolet N, Weller M, Kros J, Hainfellner J, Mason W, Mariani L, Bromberg J, Hau P, Mirimanoff R, Cairncross J, Janzer RRS (2005) MGMT gene silencing and benefit from Temozolomide in glioblastoma. N Engl J Med 352:997-1003

15. Martinez R, Schackert G, Yaya-Tur R, Rojas-Marcos I, Herman JG, Esteller M (2007) Frequent hypermethylation of the DNA repair gene MGMT in long-term survivors of glioblastoma multiforme. J Neurooncol 83:91-93

16. Stupp R, Hegi ME, Gilbert MR, Chakravarti A (2007) Chemoradiotherapy in malignant glioma: standard of care and future directions. J Clin Oncol 25:4127-4136

17. Laird PW (2003) The power and the promise of DNA methylation markers. Nat Rev Cancer 253:253-266

18. Karayan-Tapon L, Quillien V, Guihot J, Wager M, Fromont G, Saikali S, Etcheverry A, Hamlat A, Loussouarn D, Champion L, Campone M, Vallette F-M, Gratas-Rabbia-Re C (2010) Prognostic value of O6-methylguanine-DNA methyltransferase status in glioblastoma patients, assessed by five different methods. J Neurooncol 97:311-322
19. Stupp R, Hegi ME, Mason WP, van den Bent MJ, Taphoorn MJ, Janzer RC, Ludwin SK, Allgeier A, Fisher B, Belanger K, Hau P, Brandes AA, Gijtenbeek J, Marosi C, Vecht CJ, Mokhtari K, Wesseling P, Villa S, Eisenhauer E, Gorlia T, Weller M, Lacombe D, Cairncross JG, Mirimanoff RO (2009) Effects of radiotherapy with concomitant and adjuvant temozolomide versus radiotherapy alone on survival in glioblastoma in a randomised phase III study: 5-year analysis of the EORTC-NCIC trial. Lancet Oncol 10:459-466

20. Cankovic M, Mikkelsen T, Rosenblum ML, Zarbo RJ (2007) A simplified laboratory validated assay for MGMT promoter hypermethylation analysis of glioma specimens from formalin-fixed paraffin-embedded tissue. Lab Invest 87:392-397

21. Vlassenbroeck I, Califice S, Diserens AC, Migliavacca E, Straub J, Di Stefano I, Moreau F, Hamou MF, Renard I, Delorenzi M, Flamion B, DiGuiseppi J, Bierau K, Hegi ME (2008) Validation of real-time methylation-specific PCR to determine O6-methylguanine-DNA methyltransferase gene promoter methylation in glioma. J Mol Diagn 10:332-337

22. Nancarrow D, HY H, Stark M, Whiteman D, NK H (2007) SiDCoN: a tool to aid scoring of DNA copy number changes in SNP chip data. PLoS One 2:e1093

23. Herman JG, Graff JR, Myohanen S, Nelkin BD, Baylin SB (1996) Methylation-specific PCR: a novel PCR assay for methylation status of $\mathrm{CpG}$ islands. Proc Natl Acad Sci USA 93:9821-9826

24. Mikeska T, Bock C, El-Maarri O, Hubner A, Ehrentraut D, Schramm J, Felsberg J, Kahl P, Buttner R, Pietsch T, Waha A (2007) Optimization of quantitative MGMT promoter methylation analysis using pyrosequencing and combined bisulfite restriction analysis. J Mol Diagn 9:368-381

25. Hattermann K, Mehdorn HM, Mentlein R, Schultka S, Held-Feindt J (2008) A methylation-specific and SYBR-green-based quantitative polymerase chain reaction technique for O6-methylguanine DNA methyltransferase promoter methylation analysis. Anal Biochem 377:62-71

26. Gonzalgo ML, Jones PA (2002) Quantitative methylation analysis using methylation-sensitive single-nucleotide primer extension (Ms-SNuPE). Methods 27:128-133

27. Jeuken JW, Cornelissen SJ, Vriezen M, Dekkers MM, Errami A, Sijben A, Boots-Sprenger SH, Wesseling P (2007) MS-MLPA: an attractive alternative laboratory assay for robust, reliable, and semiquantitative detection of MGMT promoter hypermethylation in gliomas. Lab Invest 87:1055-1065

28. Mollemann M, Wolter M, Felsberg J, Collins VP, Reifenberger G (2005) Frequent promoter hypermethylation and low expression of the MGMT gene in oligodendroglial tumors. Int $\mathrm{J}$ Cancer 113:379-385

29. Christensen BC, Houseman EA, Marsit CJ, Zheng S, Wrensch MR, Wiemels JL, Nelson HH, Karagas MR, Padbury JF, Bueno R, Sugarbaker DJ, Yeh RF, Wiencke JK, Kelsey KT (2009) Aging and environmental exposures alter tissue-specific DNA methylation dependent upon $\mathrm{CpG}$ island context. PLoS Genet 5:e1000602

30. Fraga MF, Agrelo R, Esteller M (2007) Cross-talk between aging and cancer: the epigenetic language. Ann NY Acad Sci 1100:60-74

31. Parrella P, la Torre A, Copetti M, Valori VM, Barbano R, Notarangelo A, Bisceglia M, Gallo AP, Balsamo T, Poeta ML, Carella M, Catapano D, Parisi S, Dallapiccola B, Maiello E, D'Angelo V, Fazio VM (2009) High specificity of quantitative methylationspecific PCR analysis for MGMT promoter hypermethylation detection in gliomas. J Biomed Biotechnol 2009:531692

32. Preusser M (2009) MGMT analysis at DNA, RNA and protein levels in glioblastoma tissue. Histol Histopathol 24:511-518 
33. Weller M, Stupp R, Reifenberger G, Brandes AA, van den Bent MJ, Wick W, Hegi ME (2010) MGMT promoter methylation in malignant gliomas: ready for personalized medicine? Nat Rev Neurol 6:39-51

34. Dunn J, Baborie A, Alam F, Joyce K, Moxham M, Sibson R, Crooks D, Husband D, Shenoy A, Brodbelt A, Wong H, Liloglou T, Haylock B, Walker C (2009) Extent of MGMT promoter methylation correlates with outcome in glioblastomas given temozolomide and radiotherapy. Br J Cancer 101:124-131

35. Juillerat-Jeanneret L, Bernasconi CC, Bricod C, Gros S, Trepey S, Benhattar J, Janzer RC (2008) Heterogeneity of human glioblastoma: glutathione-S-transferase and methylguanine-methyltransferase. Cancer Invest 26:597-609

36. Parkinson JF, Wheeler HR, Clarkson A, McKenzie CA, Biggs MT, Little NS, Cook RJ, Messina M, Robinson BG, McDonald KL (2008) Variation of $\mathrm{O}(6)$-methylguanine-DNA methyltransferase (MGMT) promoter methylation in serial samples in glioblastoma. J Neurooncol 87:71-78

37. Grasbon-Frodl EM, Kreth FW, Ruiter M, Schnell O, Bise K, Felsberg J, Reifenberger G, Tonn JC, Kretzschmar HA (2007) Intratumoral homogeneity of MGMT promoter hypermethylation as demonstrated in serial stereotactic specimens from anaplastic astrocytomas and glioblastomas. Int J Cancer 121:2458-2464

38. Ochsenbein AF, Schubert AD, Vassella E, Mariani L (2010) Quantitative analysis of $\mathrm{O}(6)$-methylguanine DNA methyltransferase (MGMT) promoter methylation in patients with low-grade gliomas. J Neurooncol [Epub ahead of print]

39. van den Bent MJ, Dubbink HJ, Sanson M, van der Lee-Haarloo CR, Hegi M, Jeuken JW, Ibdaih A, Brandes AA, Taphoorn MJ, Frenay M, Lacombe D, Gorlia T, Dinjens WN, Kros JM (2009) MGMT promoter methylation is prognostic but not predictive for outcome to adjuvant PCV chemotherapy in anaplastic oligodendroglial tumors: a report from EORTC Brain Tumor Group Study 26951. J Clin Oncol 27:5881-5886

40. Wick W, Hartmann C, Engel C, Stoffels M, Felsberg J, Stockhammer F, Sabel MC, Koeppen S, Ketter R, Meyermann R, Rapp M, Meisner C, Kortmann RD, Pietsch T, Wiestler OD, Ernemann U, Bamberg M, Reifenberger G, von Deimling A, Weller M (2009) NOA-04 randomized phase III trial of sequential radiochemotherapy of anaplastic glioma with procarbazine, lomustine, and vincristine or temozolomide. J Clin Oncol 27:5874-5880 